University of Texas at El Paso

ScholarWorks@UTEP

$11-2009$

\title{
From Gauging Accuracy of Quantity Estimates to Gauging Accuracy and Resolution of Measuring Physical Fields
}

Vladik Kreinovich

The University of Texas at El Paso, vladik@utep.edu

Irina Perfilieva

Follow this and additional works at: https://scholarworks.utep.edu/cs_techrep

Part of the Computer Engineering Commons

Comments:

Technical Report: UTEP-CS-09-16c

Published in Roman Wyrzykowski, Jack Dongarra, Konrad Kzrczewski, and Jerzy Wasniewski (eds.), Proceedings of the Eighth International Conference on Parallel Processing and Applied Mathematics PPAM'2009, Wroclaw, Poland, September 13-16, 2009, Springer Lecture Notes in Computer Science, 2010, Vol. 6608, pp. 456-465.

\section{Recommended Citation}

Kreinovich, Vladik and Perfilieva, Irina, "From Gauging Accuracy of Quantity Estimates to Gauging Accuracy and Resolution of Measuring Physical Fields" (2009). Departmental Technical Reports (CS). 42. https://scholarworks.utep.edu/cs_techrep/42

This Article is brought to you for free and open access by the Computer Science at ScholarWorks@UTEP. It has been accepted for inclusion in Departmental Technical Reports (CS) by an authorized administrator of ScholarWorks@UTEP.For more information, please contact Iweber@utep.edu. 


\title{
From Gauging Accuracy of Quantity Estimates to Gauging Accuracy and Resolution of Measuring Physical Fields
}

\author{
Vladik Kreinovich ${ }^{1}$ and Irina Perfilieva ${ }^{2}$ \\ 1 University of Texas, El Paso, TX 79968, USA, vladik@utep.edu \\ 2 University of Ostrava, Inst. for Research and Applications of Fuzzy Modeling, \\ 70100 Ostrava, Czech Republic, Irina.Perfilieva@osu.cz
}

\begin{abstract}
For a numerical physical quantity $v$, because of the measurement imprecision, the measurement result $\widetilde{v}$ is, in general, different from the actual value $v$ of this quantity. Depending on what we know about the measurement uncertainty $\Delta v \stackrel{\text { def }}{=} \widetilde{v}-v$, we can use different techniques for dealing with this imprecision: probabilistic, interval, etc.

When we measure the values $v(x)$ of physical fields at different locations $x$ (and/or different moments of time), then, in addition to the same measurement uncertainty, we also encounter another type of localization uncertainty: that the measured value may come not only from the desired location $x$, but also from the nearby locations.

In this paper, we discuss how to handle this additional uncertainty.
\end{abstract}

\section{Formulation of the Problem}

Need for data processing. In many real-life situations, we are interested in the value of a quantity which is difficult (or even impossible) to measure directly. For example, we may be interested in the distance to a star, or in the amount of water in an underground water layer. Since we cannot measure the corresponding quantity $y$ directly, we measure it indirectly. Specifically,

- we find easier-to-measure quantities $x_{1}, \ldots, x_{n}$ which are related to the desired quantity $y$ by a known dependence $y=f\left(x_{1}, \ldots, x_{n}\right)$;

- we measure the values of the auxiliary quantities $x_{1}, \ldots, x_{n}$; and

- we use the results $\widetilde{x}_{1}, \ldots, \widetilde{x}_{n}$ of measuring the auxiliary quantity to compute the estimate $\widetilde{y}=f\left(\widetilde{x}_{1}, \ldots, \widetilde{x}_{n}\right)$ for the desired quantity $y$.

Example. To find the distance $y$ to a star, we can use the following parallax method:

- we measure the orientations $x_{1}$ and $x_{2}$ to this star at two different seasons,

- we measure the the distance $x_{3}$ between the spatial locations of the corresponding telescopes at these two seasons (i.e., in effect, the diameter of the earth orbit);

- then, reasonably simply trigonometric computations enable us to describe the desired distance $y$ as a function of the easier-to-measure quantities $x_{1}$, $x_{2}$, and $x_{3}$. 
General case. In general, computations related to such indirect measurements form an important particular case of data processing.

Need to take uncertainty into account. Measurements are never absolutely accurate. As a result, the measurement results $\widetilde{x}_{i}$ are, in general, different from the actual (unknown) values $x_{i}$ of the measured quantities: $\Delta x_{i} \stackrel{\text { def }}{=} \widetilde{x}_{i}-x_{i} \neq 0$. Because of this, the result $\widetilde{y}=f\left(\widetilde{x}_{1}, \ldots, \widetilde{x}_{n}\right)$ of data processing is, in general, different from the actual (unknown) value $y=f\left(x_{1}, \ldots, x_{n}\right): \Delta y \stackrel{\text { def }}{=} \widetilde{y}-y \neq 0$.

Thus, in practical applications, we need to take this uncertainty into account.

Interval uncertainty. In practice, we often only know the upper bound $\Delta_{i}$ on the measurement errors $\Delta x_{i} \stackrel{\text { def }}{=} \widetilde{x}_{i}-x_{i}:\left|\Delta x_{i}\right| \leq \Delta_{i}$. In this case, the only information that we have about the actual values $x_{i}$ is that $x_{i}$ belongs to the interval $\mathbf{x}_{i} \stackrel{\text { def }}{=}\left[\widetilde{x}_{i}-\Delta_{i}, \widetilde{x}_{i}+\Delta_{i}\right]$.

Under such interval uncertainty, we need to find the range of possible values of $y: \mathbf{y}=\left\{f\left(x_{1}, \ldots, x_{n}\right): x_{i} \in \mathbf{x}_{i}\right\}$. The problem of computing this range is known as interval computations; see, e.g., [4].

Need to measure physical fields. In practice, the situation is often more complex: the values that we measure can be:

- values $v(t)$ of a certain dynamic quantity $v$ at a certain moment of time $t$

- or, more generally, the values $v(x, t)$ of a certain physical field $v$ at a certain location $x$ and at a certain moment of time $t$.

For example, in geophysics, we are interested in the values of the density at different locations and at different depth.

Need to take uncertainty into account when measuring physical fields. When we measure physical fields, not only we get the measured value $\widetilde{v} \approx v$ with some inaccuracy, but also the location $x$ is not exactly known. Moreover, the sensor picks up the "averaged" value of $v$ at locations close to the approximately known location $\widetilde{x}$. In other words, in addition to inaccuracy $\widetilde{v} \neq v$, we also have a finite (spatial) resolution $\widetilde{x} \neq x$.

Estimating uncertainty related to measuring physical fields: challenging problems. In general, the measured value $\widetilde{v}_{i}$ differs from the averaged value $v_{i}$ by the measurement imprecision $\Delta v_{i}=\widetilde{v}_{i}-v_{i}$. In the interval case, we know the upper bound $\Delta_{i}$ on this measurement error $\left|\Delta v_{i}\right| \leq \Delta_{i}$. Thus, the averaged quantity $v_{i}$ can take any value from the interval $\left[\underline{v}_{i}, \bar{v}_{i}\right]$, where $\underline{v}_{i} \stackrel{\text { def }}{=} \widetilde{v}_{i}-\Delta_{i}$ and $\bar{v}_{i} \stackrel{\text { def }}{=} \widetilde{v}_{i}+\Delta_{i}$.

Based on these bounds on $v_{i}$, what can we learn about the original field $v(x)$ ? The answer to this questions depends on what we know about the averaging, i.e., on the dependence of $v_{i}$ on $v(x)$. In principle, there are three possible situations:

- sometimes, we know exactly how the averaged values $v_{i}$ are related to $v(x)$; 
- sometimes, we only know the upper bound $\delta$ on the location error $\widetilde{x}-x$ (this is similar to the interval case);

- sometimes, we do not even know $\delta$.

In the following sections, we describe how to process all these types of uncertainty.

\section{Possibility of Linearization}

Sometimes, the signal $v(x)$ that we are measuring is large, i.e., the values of the signal are much larger than the noise (and the measurement errors in general). In such situations, the measured values well represent the actual signal, and for many applications, the measurement errors can be safely ignored.

The need to take into account measurement errors becomes important only when the signal $v(x)$ is relatively weak. In this case, we can expand the dependence of $v_{i}$ on $v(x)$ in Taylor series. To describe this expansion, let us first consider a simplified case in which there are only finitely many spatial points $x^{(1)}$, $\ldots, x^{(N)}$, so the field $v(x)$ is described by finitely many values $v^{(j)} \stackrel{\text { def }}{=} v\left(x^{(j)}\right)$, $j=1, \ldots, N$. In this case, the dependence of the quantity $v_{i}$ on these values $v^{(1)}, \ldots, v^{(N)}$ can be expanded into Taylor series

$$
v_{i}=a_{i}+\sum_{j=1}^{N} a_{i j} \cdot v\left(x^{(j)}\right)+\sum_{j=1}^{N} \sum_{j=k}^{N} a_{i j k} \cdot v\left(x^{(j)}\right) \cdot v\left(x^{(k)}\right)+\ldots
$$

The description in terms of finitely many spatial points is an approximate description of the actual field $v(x)$. The more points we consider and the denser they are located in the domain $D$ on which this field is defined, the more accurate the corresponding approximation. In the limit, when the distance between the points tends to 0 (and the approximation accuracy tends to 0 ), the sums in (1) turn into integrals, so the formula (1) takes the form

$$
v_{i}=a_{i}+\int_{D} w_{i}(x) \cdot v(x) d x+\int_{D \times D} w_{i}\left(x, x^{\prime}\right) \cdot v(x) \cdot v\left(x^{\prime}\right) d x d x^{\prime}+\ldots
$$

for appropriate functions $w_{i}(x), w_{i}\left(x, x^{\prime}\right)$, etc.

Since the signal $v(x)$ is relatively weak, we can safely ignore quadratic and higher order terms in this dependence. Also, we know that in the absence of the field, when $v(x)=0$, the differences $v_{i}$ are $0 \mathrm{~s}$, so we have $a_{i}=0$. As a result, we get a linear expression for $v_{i}$ in terms of $v(x): v_{i}=\int_{D} w_{i}(x) \cdot v(x) d x$.

\section{Case of Full Information about the Resolution}

Description. In this section, we consider the case when we know the exact expression for this dependence, i.e., when we know the weights $w_{i}(x)$. 
The notion of fuzzy transform. Intuitively, each "averaged" value $v_{i}$ can be viewed as the value of the field $v(x)$ at a "fuzzy" point characterized by uncertainty $w_{i}(x)$. Because of this interpretation, the transformation from the original function $v(x)$ to the set of values $v_{1}, \ldots, v_{n}$ is also known as a fuzzy transform; see, e.g., $[6,7]$.

Comment. From the physical viewpoints, the weights $w_{i}(x)$ are not probabilities. However, since probability theory is the oldest - and most developed formalism for describing uncertainty, it is not surprising that often, computational techniques from probability theory can be efficiently used to described other types of uncertainty as well. In particular, in the following text, we will see that for our problem, techniques from imprecise probability theory can be very useful.

What we want to predict. Based on the measurement results $\widetilde{v}_{1}, \ldots, \widetilde{v}_{n}$, we would like to reconstruct the field $v(x)$. From the pragmatic viewpoint, knowing the field means being able to predict the results of all other measurements of this field.

Each such measurement can be characterized by its own averaging function $w(x)$. Thus, predicting the result of the measurement means predicting the corresponding averaged value $y=\int_{D} w(x) \cdot v(x) d x$.

Of course, the space of functions is infinite-dimensional, which means that to uniquely reconstruct a function, we need to know infinitely many parameters. Thus, based on $n$ numbers $\widetilde{v}_{1}, \ldots, \widetilde{v}_{n}$, we cannot uniquely reconstruct the function $v(x)$ - and thus, we cannot uniquely reconstruct the desired averaged value $y$. So, the problem is to find the range $[\underline{y}, \bar{y}]$ of this value $y$.

Prediction problem as a particular case of (infinite-dimensional) linear programming (LP). The lower endpoint $y$ is the smallest possible value of $y$, the upper endpoint $\bar{y}$ is the largest possible value of $y$. Thus, the problems of finding the desired endpoints $\underline{y}$ and $\bar{y}$ can be formulated in the following optimization form: minimize (maximize) $y-\int_{D} w(x) \cdot v(x) d x$ under the constraints

$$
\underline{v}_{i} \leq \int_{D} w_{i}(x) \cdot v(x) d x \leq \bar{v}_{i}, \quad 1 \leq i \leq n
$$

In both problems, we optimize the value of a linear functional under linear constraints. In the finite-dimensional case, when we have finitely many unknowns, such constraint optimization problems are known as linear programming (LP) problems. In our case, an unknown is a function $v(x)$, and the linear space of all possible functions is infinite-dimensional. Thus, from the mathematical viewpoint, our problems are infinite-dimensional analogues of linear programming (LP) problems; see, e.g., [1].

Without prior restrictions on the field $v(x)$, we cannot predict anything. In general, if we do not impose any conditions on the function $v(x)$, then both bounds 
are infinite - unless $w(x)$ is a linear combination of $w_{i}(x)$. Indeed, it is known that every vector $w$ which is orthogonal to all the vectors $t$, which are orthogonal to all the vectors $w_{1}, \ldots, w_{n}$, belongs to the linear space generated by the vectors $w_{1}, \ldots, w_{n}$-i.e., is a linear combination of $w_{1}, \ldots, w_{n}$. Thus, if a vector $w$ cannot be represented as a linear combination of the vectors $w_{1}, \ldots, w_{n}$, then there exists a vector $t$ which is orthogonal to all $w_{i}$ but not to $w$. With respect to the space of all the functions, this means that if $w(x)$ cannot be represented as a linear combination of the functions $w_{i}(x)$, then there exists a function $t(x)$ which is orthogonal to all $w_{i}(x)$ (in the sense that $\left\langle w_{i}, t\right\rangle \stackrel{\text { def }}{=} \int_{D} w_{i}(x) \cdot t(x) d x=0$ ) but not to $w(x)(\langle w, t\rangle \neq 0)$.

For an arbitrary real number $\lambda$, instead of the actual field $v(x)$, we can now consider a new field $v_{\lambda}(x) \stackrel{\text { def }}{=} v(x)+\lambda \cdot t(x)$. For this new field $v_{\lambda}(x)$, the values of $v_{i}$ are the same as for the original field $v(x)$ - and hence, satisfy the same inequalities. However, the new value $y$ is equal to $y_{\lambda}=\langle w, v\rangle+\lambda \cdot\langle w, t\rangle$. Since $\langle w, t\rangle \neq 0$, for appropriate $\lambda$, we can get this value $y_{\lambda}$ equal to any given real number. Thus, indeed, the smallest possible value of $y$ is $y=-\infty$, and the largest possible value of $y$ is $\bar{y}=+\infty$.

Non-negative fields. In many practical problems, the field $v(x)$ can only have non-negative values $v(x) \geq 0$. For example, in geophysics, the density $v(x)$ cannot be negative. Under this additional restrictions, we already have non-trivial bounds $y$ and $\bar{y}$.

Dual LP techniques. For solving these problems, we can use the experience of imprecise probabilities $[5,9]$ where we have similar LP problems. In these problems, the unknown function $v(x)$ is the non-negative probability density function (pdf), and the observed values have the same form

$$
v_{i}=\int_{D} w_{i}(x) \cdot x(x) d x
$$

For example, the second moment of the probability distribution with the pdf $v(x)$ has the form $\int_{D} x^{2} \cdot v(x) d x$, so it has the desired form with the weight function $w_{i}(x)=x^{2}$.

In the imprecise probability theory, it is reasonable to ask what is, e.g., the interval of possible values of the third moment if we know values of (or bounds on) the first and the second moments. In mathematical terms, we thus arrive at the same infinite-dimensional LP problems as in our measurement cases.

According to the experience of imprecise probabilities, many efficient algorithms for solving the corresponding LP problems come from considering dual LP problems, i.e., by computing the range $[\underline{v}, \bar{v}]$, where

$$
\underline{v}=\sup \left\{\sum y_{i} \cdot \underline{v}_{i}: \sum y_{i} \cdot w_{i}(x) \leq w(x)\right\} ;
$$

and $\bar{v}=\inf \left\{\sum y_{i} \cdot \bar{v}_{i}: w(x) \leq \sum y_{i} \cdot w_{i}(x)\right\}$. 
Indeed, if $\sum y_{i} \cdot w_{i}(x) \leq w(x)$, then, by multiplying both sides of this inequality by $v(x) \geq 0$ and integrating over $x$, we conclude that $\sum y_{i} \cdot v_{i} \leq y$. Since we know that $v_{i} \geq \underline{v}_{i}$, we thus get a lower bound for $y: y \geq \sum y_{i} \cdot \underline{v}_{i}$. Thus, $y$ is larger than the largest of these bounds, i.e., $y \geq \underline{v}$. So, we can conclude that $\underline{y} \geq \underline{v}$. Similarly, we can conclude that $\bar{y} \leq \bar{v}$, i.e., that the dual LP interval $[\underline{v}, \bar{v}]$ is the enclosure for the desired range $[y, \bar{y}]$.

\section{Comments.}

- For finite-dimensional LP problems, the dual interval is exactly equal to the original one.

- Our problems are easier than the imprecise probability ones, since the functions $w_{i}(x)$ are usually localized and thus, for each $x$, usually at most a few functions $w_{i}(x)$ differ from 0 . This makes checking the sums easier.

- Checking the inequalities like $\sum y_{i} \cdot w_{i}(x) \leq w(x)$ is even easier in a practically important case of piece-wise linear functions $w_{i}(x)$ and $w(x)$. In this case, it is sufficient to check this inequality at endpoints of linearity intervals - then, due to linearity, it will be automatically true for all internal points as well.

\section{Situations in Which We Only Know Upper Bounds}

General idea. In other cases - similarly to the interval setting - we do not only know the upper bounds $\delta$ on the location error $\widetilde{x}-x$. A natural question is: when is a model $v(x)$ consistent with the given observation $(\widetilde{v}, \widetilde{x}) ?$

In this case, the measured value $\widetilde{v}$ is $\Delta$-close to a convex combination of values $v(x)$ for $x$ s.t. $\|x-\widetilde{x}\| \leq \Delta x$. Thus, $\underline{v}_{\delta}(\widetilde{x})-\Delta \leq \widetilde{v} \leq \bar{v}_{\delta}(\widetilde{x})+\Delta$, where:

$$
\underline{v}_{\delta}(\widetilde{x}) \stackrel{\text { def }}{=} \inf \{v(x):\|x-\widetilde{x}\| \leq \delta\}, \text { and } \bar{v}_{\delta}(\widetilde{x}) \stackrel{\text { def }}{=} \sup \{v(x):\|x-\widetilde{x}\| \leq \delta\} .
$$

Case of interval models. In real life, we rarely have an exact model $v(x)$. Usually, we have bounds on $v(x)$, i.e., an interval-valued model $\mathbf{v}(x)=\left[v^{-}(x), v^{+}(x)\right]$. An observation $(\widetilde{v}, \widetilde{x})$ consistent with this "interval-valued" model if there exists a model $v(x) \in \mathbf{v}(x)$ which is consistent with this observation.

Since the values $\underline{v}_{\delta}$ and $\bar{v}_{\delta}$ monotonically depend on $v(x)$, this consistency leads to $\underline{v}_{\delta}^{-}(\widetilde{x})-\Delta \leq \widetilde{v} \leq \bar{v}_{\delta}^{+}(\widetilde{x})+\Delta$.

Relation to Hausdorff metric. In many practical problems, the field $v(x)$ continuously depends on $x$. For continuous functions, inf and sup on a bounded closed set $\{x:\|x-\widetilde{x}\| \leq \delta\}$ are attained at some value. Thus, the above criterion for consistency between a model and observations can be simplified.

Namely, in this case, the set $\widetilde{m}$ of all measurement results $(\widetilde{v}, \widetilde{x})$ is consistent with the model $v(x)$ if and only if

$$
\forall(\widetilde{v}, \widetilde{x}) \in \widetilde{m} \exists(v(x), x) \in v^{-1}((\widetilde{v}, \widetilde{x}) \text { is }(\Delta, \delta) \text {-close to }(v(x), x)),
$$


i.e., $|\widetilde{v}-v| \leq \Delta$ and $\|x-\widetilde{x}\| \leq \delta$. In this formula, $v^{-1} \stackrel{\text { def }}{=}\{(v(x), x): x \in D\}$, i.e., $v^{-1}$ is the inverse relation to the relation $v=\{(x, v(x)): x \in D\}$ describing the function $v(x)$.

The notion of $(\Delta, \delta)$-closeness between points $(v, x)$ and $\left(v^{\prime}, x^{\prime}\right)$ can be formally described as $d\left((v, x),\left(v^{\prime}, x^{\prime}\right)\right) \leq(\Delta, \delta)$, where

$d\left((v, x),\left(v^{\prime}, x^{\prime}\right)\right) \stackrel{\text { def }}{=}\left(\left|v-v^{\prime}\right|,\left\|x-x^{\prime}\right\|\right) ; \quad(\Delta, \delta) \leq\left(\Delta^{\prime}, \delta^{\prime}\right) \Leftrightarrow\left(\left(\Delta \leq \Delta^{\prime}\right) \&\left(\delta \leq \delta^{\prime}\right)\right)$.

This definition is similar to the standard definition of the Hausdorff metric $d_{H}$ : $d_{H}(A, B) \leq \varepsilon$ means that

$$
\forall a \in A \exists b \in B(d(a, b) \leq \varepsilon) \text { and } \forall b \in B \exists a \in A(d(a, b) \leq \varepsilon) .
$$

(This similarity was noticed in [2].)

Specifically, the above definition is an asymmetric two-dimensional version of Hausdorff metric. Let us show, on a simple example, that our "distance" is indeed asymmetric.

Case 1:

In this example,

- the actual field has the form $v(0)=1$ and $v(x)=0$ for $x \neq 0$, and

- the measurements results are all zeros, i.e., $\widetilde{v}=0$ for all $\widetilde{x}$.

In this case, all the measurements are consistent with the model:

- the values $\widetilde{v}=0$ for $\widetilde{x} \neq 0$ are consistent with $v=0$ for $x=\widetilde{x}$, and

- the value $\widetilde{v}=0$ for $\widetilde{x}=0$ is consistent with $v(x)=0$ for $x=\delta$ s.t. $|\widetilde{x}-x| \leq \delta$.

Case 2:

In this example,

- the actual field is all zeros, i.e., $v(x)=0$ for all $x$, and

- the measurement results are $\widetilde{v}=1$ for $\widetilde{x}=0$, and $\widetilde{v}=0$ for all $\widetilde{x} \neq 0$.

Here, when $\Delta<1$, the measurement $(1,0)$ is inconsistent with the model, because for all $x$ which are $\delta$-close to $\widetilde{x}=0$, we have $v(x)=0$ hence we should have $|\widetilde{x}-v(x)|=|\widetilde{x}| \leq \Delta$.

\section{Case of Minimal Knowledge About Uncertainty}

Idea. Yet another case is when we do not even know $\delta$. It happened, e.g., when we solve the seismic inverse problem to find the velocity distribution. In this case, a natural heuristic idea is:

- to add a perturbation of size $\delta_{0}$ (e.g., sinusoidal) to the reconstructed field $\widetilde{v}(x)$, 
- to simulate the new measurement results,

- to apply the same algorithm to the simulated results, and

- to reconstruct the new field $\widetilde{v}_{\text {new }}(x)$.

If the perturbations are not visible in $\widetilde{v}_{\text {new }}(x)-\widetilde{v}(x)$, this means that details of size $\delta_{0}$ cannot be reconstructed and so, the actual resolution is $\delta>\delta_{0}$. This approach was partially described in $[3,8]$.

Linearization and its consequences. Which perturbations should we choose? To select the optimal perturbations, we will take into account the fact that since perturbations are usually small, we can safely linearize their effects. Thus, if we know the results $\Delta v_{1}(x), \ldots, \Delta v_{k}(x)$ of applying perturbations $e_{1}(x), \ldots, e_{k}(x)$, we can predict the result $\Delta v(x)$ of applying a linear combination

$$
e(x)=c_{1} \cdot e_{1}(x)+\ldots+c_{k} \cdot e_{k}(x),
$$

as $\Delta v(x)=c_{1} \cdot \Delta v_{1}(x)+\ldots+c_{k} \cdot \Delta v_{k}(x)$. In other words, once we know the results of applying $k$ different perturbations $e_{1}(x), \ldots, e_{k}(x)$, we thus also know the results of applying an arbitrary perturbation from the linear space $L=$ $\left\{c_{1} \cdot e_{1}(x)+\ldots+c_{k} \cdot e_{k}(x)\right\}$. From this viewpoint, it does not matter what exactly perturbations $e_{i}(x)$ we select as long as they are within the same space $L$.

Thus, the question of optimally selecting a given number $k$ of perturbations can be formulated as the question of optimally selecting a $k$-dimensional linear subspace $L$ in the space of all functions.

Shift-invariance: a natural requirement. To select the space $L$, let us use the fact that in most physical problems, there is no preferred spatial location. Thus, in principle, we can choose different locations as origins $(x=0)$ of the coordinate system.

It is reasonable to require that the optimal family of perturbations do not change if we simply change the origin $x=0$. For example, if we select a point with the original coordinates $x_{0}$ as the origin of a new coordinate system, then the new coordinates will have the form $x_{\text {new }}=x-x_{0}$. In the original coordinates, the optimal family of perturbations has the form $\left\{c_{1} \cdot e_{1}(x)+\ldots+c_{k} \cdot e_{k}(x)\right\}$. In the new coordinates $x_{\text {new }}$, we should expect the exact same family of perturbations $\left\{c_{1} \cdot e_{1}\left(x_{\text {new }}\right)+\ldots+c_{k} \cdot e_{k}\left(x_{\text {new }}\right)\right\}$. In terms of the original coordinates, this new family has the form $\left\{c_{1} \cdot e_{1}\left(x-x_{0}\right)+\ldots+c_{k} \cdot e_{k}\left(x-x_{0}\right)\right\}$.

This "shifted" family must coincide with the original one. In particular, every basis function $e_{i}\left(x-x_{0}\right)$ from the shifted basis must belong to the original family, i.e., must have the form $e_{i}\left(x+x_{0}\right)=\sum_{j=1}^{k} c_{i j}\left(x_{0}\right) \cdot e_{j}(x)$ for some coefficients $c_{i j}$ which are, in general, depending on the shift $x_{0}$.

Smoothness: an additional requirement. In many physical problems, it is reasonable to consider smooth perturbations, i.e., perturbations for which the functions $e_{i}(x)$ are differentiable. In this case, by considering different values $x$, we get a 
system of linear equations for determining $c_{i j}\left(x_{0}\right)$ in terms of the smooth functions $e_{i}\left(x+x_{0}\right)$ and $e_{j}(x)$. The solution of a system of linear equations is - due to Cramer's rule - a smooth function of the coefficients and of the right-hand sides. Thus, the solutions $c_{i j}\left(x_{0}\right)$ are also smooth.

From the requirements to the description of the desired family $L$. Let us fix one of the spatial coordinates, e.g., the coordinate $x_{1}$. For shifts w.r.t. this coordinate, we have $e_{i}\left(x_{1}+x_{0}, x_{2}, \ldots\right)=\sum_{j=1}^{k} c_{i j}\left(x_{0}\right) \cdot e_{j}\left(x_{1}, x_{2}, \ldots\right)$

Since the functions $e_{i}\left(x_{1}+x_{0}, \ldots\right)$ and $c_{i j}\left(x_{0}\right)$ are smooth, we can differentiate both sides of the above equation with respect to $x_{0}$ and take $x_{0}=0$. For each components of $x_{0}$, we get a system of linear differential equations $e_{i}^{\prime}=\sum c_{i j}^{\prime}(0) \cdot e_{j}$ with constant coefficients. A general solution to such a system is well known: it is a linear combination of expressions $x_{1}^{k_{1 j}} \cdot \exp \left(a_{1 j} \cdot x_{1}\right)$ with complex values $a_{1 j}$ (eigenvalues of the matrix $c_{i j}^{\prime}(0)$ ) and integers $k_{1 j} \geq 0$ (multiplicities of these eigenvalues).

Some of these solutions tend to infinity exponentially fast. Such solutions are not useful as perturbations, since perturbations must be uniformly small. So, it is reasonable to restrict ourselves to bounded perturbations.

This boundedness eliminates the terms with $\operatorname{Re}\left(a_{1 j}\right) \neq 0$. Thus, the only remaining terms correspond to imaginary values $a_{1 j}$ - i.e., to sinusoids. For these terms, boundedness also eliminates terms with $k_{1 j}>0$, so we only get pure sinusoids: $e_{i}\left(x_{1}, x_{2}, \ldots\right)=\sum_{j} C_{j}\left(x_{2}, \ldots\right) \cdot \sin \left(\omega_{1 j} \cdot x_{1}\right)$. The functions $C_{j}\left(x_{2}, \ldots\right)$ can be computed as linear combinations of the values $e_{i}\left(x_{1}, x_{2}, \ldots\right)$ corresponding to different values $x_{1}$. On the other hand, the dependence of $e_{i}$ on $x_{2}$ is also a linear combination of sinusoids. Thus, the functions $C_{j}\left(x_{2}, \ldots\right)$ are linear combinations of sinusoids in $x_{2}$. Substituting these linear combinations instead of $C_{j}\left(x_{2}, \ldots\right)$ into the above formula, and taking into account that $\sin (a) \cdot \sin (b)$ is a linear combination of $\cos (a+b)$ and $\cos (a-b)$, we conclude that the dependence of $e_{i}$ on $x_{1}$ and $x_{2}$ takes the form

$$
e_{i}\left(x_{1}, x_{2}, x_{3}, \ldots\right)=\sum_{k} C_{k}\left(x_{3}, \ldots\right) \cdot \sin \left(\omega_{1 k} \cdot x_{1}+\omega_{2 k} \cdot x_{2}\right)
$$

Similarly, we can add $x_{3}$, etc., and conclude that each function $e_{i}(x)$ is a linear combination of the $\operatorname{sinusoids} \sin \left(\sum \omega_{j} \cdot x_{j}+\varphi\right)$.

Resulting recommendation. We conclude that the optimal perturbations are linear combinations of sinusoids. We thus arrive at the following recommendation: to find the spatial resolution $\delta$ with which we can reconstruct the field $v(x)$, add a sinusoid with spatial wavelength $\delta_{0}$ to the reconstructed field $\widetilde{v}(x)$, simulate the new measurement result, reconstruct the new field $\widetilde{v}_{\text {new }}(x)$, and see if the perturbations are visible in $\widetilde{v}_{\text {new }}(x)-\widetilde{v}(x)$. 


\section{Conclusions}

When we measure the values $v(x)$ of physical fields at different locations $x$ (and/or different moments of time), then, in addition to the measurement uncertainty, we also encounter another type of localization uncertainty: that the measured value may come not only from the desired location $x$, but also from the nearby locations. In this paper, we discuss how to handle this additional uncertainty. Specifically:

- in situations in which we know the exact dependence of the measured value on the field $v(x)$, we can use infinite-dimensional versions of linear programming techniques;

- in situations in which we only know upper bounds on localization errors, we can use 2-dimensional versions of Hausdorff metric; and

- in situations in which we have no information about localization uncertainty, we can use sinusoidal perturbations to acquire this information.

Acknowledgments. This work was supported in part by the National Science Foundation grant HRD-0734825, by Grant 1 T36 GM078000-01 from the National Institutes of Health, and by Grant MSM 6198898701 from MŠMT of Czech Republic. The authors are thankful to the anonymous referees and to the participants of PPAM'09 for valuable suggestions.

\section{References}

1. E. J. Anderson and P. Nash, Linear Programming in Infinite-Dimensional Spaces, Wiley, New York, 1987.

2. R. Anguelov, S. Markov, and B. Sendov, "The set of Hausdorff continuous functions - the largest linear space of interval functions", Reliable Computing, 2006, Vol. 12, pp. 337-363.

3. M. G. Averill, A Lithospheric Investigation of the Southern Rio Grande Rift, PhD Dissertation, University of Texas at El Paso, Department of Geological Sciences, 2007.

4. L. Jaulin et al., Applied Interval Analysis, Springer-Verlag, London, 2001.

5. V. Kuznetsov, Interval Statistical Methods, Radio i Svyaz Publ. Moscow, 1991 (in Russian).

6. I. Perfilieva, "Fuzzy transforms: theory and applications", Fuzzy Sets and Systems, 2006, Vol. 157, pp. 993-1023.

7. I. Perfilieva, V. Novák, and A. Dvorák, "Fuzzy transform in the analysis of data", International Journal of Approximate Reasoning, 2008, Vol. 48, No. 1, pp. 36-46.

8. P. Pinheiro da Silva et al., "Propagation and Provenance of Probabilistic and Interval Uncertainty in Cyberinfrastructure-Related Data Processing and Data Fusion", In: R. L. Muhanna and R. L. Mullen (eds.), Proceedings of the International Workshop on Reliable Engineering Computing REC'08, Savannah, Georgia, February 2022, 2008, pp. 199-234.

9. P. Walley, Statistical Reasoning with Imprecise Probabilities, Wiley, 1991. 